\title{
Spectral and polarization properties of reflected X-ray emission from black hole accretion discs
}

\author{
J. Podgorný1 ${ }^{1,2,3 \star}$, M. Dovčiak ${ }^{2}$, F. Marin ${ }^{1}$, R. Goosmann ${ }^{1}$ and A. Różańska ${ }^{4}$ \\ ${ }^{1}$ Université de Strasbourg, CNRS, Observatoire Astronomique de Strasbourg, UMR 7550, F-67000 Strasbourg, France \\ ${ }^{2}$ Astronomical Institute, Academy of Sciences of the Czech Republic, Bočni II, CZ-14131 Prague, Czech Republic \\ ${ }^{3}$ Astronomical Institute, Charles University, V Holešovičkách 2, CZ-18000 Prague, Czech Republic \\ ${ }^{4}$ Nicolaus Copernicus Astronomical Center, Polish Academy of Sciences, Bartycka 18, 00-716 Warsaw, Poland
}

Accepted 2021 December 16. Received 2021 November 20; in original form 2021 October 8

\begin{abstract}
X-ray polarimetric missions planned for this decade will significantly enhance our knowledge of compact accreting sources. Observations of the X-ray polarization signal from active galactic nuclei (AGNs) or X-ray binary systems (XRBs) will bring new means to study inner accretion flow in these objects that, together with currently used spectroscopic and timing techniques, will help us to determine better their properties, such as their inclination, orientation, shape, and size of their corona as well as the black hole spin. In this work, we present a yet missing piece in the global polarization models of black hole accretion discs. We compute the reflected X-ray emission from the disc in a local co-moving frame using (1) the radiative transfer code TITAN to obtain the ionization structure of the disc and (2) the Monte Carlo code STOKES that incorporates the physics of absorption, re-emission, and Compton scattering to produce a complete spectropolarimetric output. We present the final Stokes parameters $I, Q$ and $U$ for a set of photon-indices of the incident primary power-law radiation, the disc ionization parameters, incident and emission angles, for three independent polarization states of the incident coronal X-ray photons with a sufficient resolution in energy to allow for sharp discussion of spectral and polarization properties. We show that the spectral component matches well literature predictions. The polarization degree and angle are in agreement with analytical approximations previously appearing in reflection models and we demonstrate that the polarized reflected X-ray emission can be, locally, quite large in the $2-12 \mathrm{keV}$ band.
\end{abstract}

Key words: accretion, accretion discs - black hole physics - polarization - radiative transfer - relativistic processes - scattering.

\section{INTRODUCTION}

Spectroscopic X-ray observations from the past decades has led to a better understanding of compact accreting sources, such as black holes (BHs) or neutron stars (NSs). These objects gradually attract matter from their surroundings which results in the formation of an accretion disc that produces tremendous amounts of light, in particular in the X-ray band (see e.g. Seward \& Charles 2010). Although these high-energy processes are considered quite omnipresent both in our Galaxy (in X-ray binary systems - XRBs) and in the rest of the Universe (in extragalactic sources such as active galactic nuclei - AGNs), a full understanding of their observed X-ray spectra is until today a difficult task. Considering mainly inner workings in radio quiet systems, i.e. excluding the presence of jets and the outer components of accreting systems, the central engine itself still poses important challenges to radiative transfer modelling in the vicinity of strongly gravitating stellar-mass objects and supermassive BHs (Antonucci 1993; Done et al. 2007; Trümper \& Hasinger 2008; Seward \& Charles 2010; Abramowicz \& Fragile 2013; Netzer 2015).

Re-opening of the X-ray polarimetric sky with the new generation, broad-band X-ray polarimeter on board of the IXPE mission (Weis-

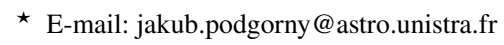

skopf et al. 2013, 2016), due to be launched by the end of 2021, or the eXTP mission (Zhang et al. 2016, 2019), due to be launched in the second half of 2020s, will help us to solve some of the current problems. The forthcoming polarimetric measurements of X-ray sources urge for (re)investigation of polarization models, in addition to plain spectral modelling. For example, the energy dependence of $\mathrm{X}$-ray polarization can provide an estimate of the BH spin (Connors \& Stark 1977; Stark \& Connors 1977; Connors et al. 1980; Dovčiak et al. 2008; Schnittman \& Krolik 2009; Li et al. 2009; Taverna et al. $2020 \mathrm{a}, \mathrm{b})$, in addition to the well-known spectroscopic techniques (using either the iron $\mathrm{K} \alpha$ line profile or the thermal disc continuum emission) or timing (kHz QPOs) techniques (see Reynolds 2019, and references therein). The sensitivity of polarization to the geometry of sources allows to investigate also other global parameters of the system, such as the system overall inclination and orientation on the sky of the observer (Dovčiak et al. 2004b, 2008; Li et al. 2009; Dovčiak et al. 2011; Marin 2014, 2016). Thus, together with the recent rise of observational X-ray polarimetry, detailed computations of X-ray emission from compact accreting sources could provide a big step towards correct data interpretation in this energy band and towards a better understanding of the high-energy Universe.

In this article, we re-examine the spectral and polarization properties of the reflected X-ray emission from AGN discs. To do so, we 
construct a numerical model that follows the general consensus of Xray emission: a power-law X-ray continuum component representing emission from a hot patch of gas -- the corona -- situated above the accretion disc (Haardt 1993; Haardt \& Maraschi 1993; Dove et al. 1997; Krolik 1999; Seward \& Charles 2010). Such $\sim E^{-\Gamma}$, powerlaw emission, where $E$ is photon energy and $\Gamma$ is photon index, is due to the reprocessing of thermal radiation by Compton up-scattering processes. This primary radiation is then partially reflected by matter in the accretion disc mostly via Compton down-scattering and thus forms a secondary X-ray component that may reach the observer with distinct spectral and polarization signatures. In the case of AGNs, where the disc temperatures are not expected to produce significant amount of thermal radiation in the X-ray band, especially in the energy band above $1 \mathrm{keV}$ (Shakura \& Sunyaev 1973; Novikov \& Thorne 1973; Reynolds \& Nowak 2003; Abramowicz \& Fragile 2013; Compère \& Oliveri 2017; Kubota \& Done 2018), the reflection tables, computed in a local fluid frame, should already realistically complement the models of primary radiation. The primary and reflected emission altogether with general-relativistic effects already comprise realistic total X-ray models of AGNs observed from infinity with the absence of jets and the absence of further radiation reprocessing via outer components, such as the broad-line regions (BLRs), narrow-line regions (NLRs), or a dusty torus (see e.g. Matt et al. 1993; Fabian et al. 2000; Seward \& Charles 2010; Netzer 2015; Kubota \& Done 2018). Although in the case of XRBs, it is necessary to account for an extra thermal component of the X-ray radiation originating from the disc (Różańska et al. 2011), the local reflection models presented here may also serve as a basic constituent for production of the total X-ray emission models of accreting stellar-mass compact objects.

Numerical attempts to compute the reflected X-ray spectrum under a power-law illumination of a disc were done most notably in the past with the codes PEXRAV and PEXRIV (Magdziarz \& Zdziarski 1995), and REFLIONX model (Ross \& Fabian 1993; Ross et al. 1999; Ross \& Fabian 2005), and more recently with the XILLVER model (García \& Kallman 2010; García et al. 2011; García et al. 2013)), including a detail computation of ionization structure of the disc by the code XSTAR (Kallman \& Bautista 2001). All the above computations assumed the reflection from a constant density slab, while stratified atmosphere in hydrostatic equilibrium was also calculated with TITAN and NOAR code (Różańska et al. 2002; Dumont et al. 2003), and with ATM24 code (Różańska \& Madej 2008; Różańska et al. 2011; Vincent et al. 2016). The most precise X-ray polarization reflection models until today consist of only analytical approximations of scattering processes and are scarce. Attempts that were able to provide estimates on the polarization degree and angle of the reflected radiation from ionized accretion disc were presented in Dovčiak et al. (2004b); Schnittman \& Krolik (2009); Dovčiak et al. (2011), assuming the Chandrasekhar's formulae (Chandrasekhar 1960) for scattering. In this article, we present the first attempt to numerically simulate both the spectral and polarization outcome of local X-ray reflection with respect to various incident and emergent angles, ionization parameters, photon-indices and polarization states of the primary radiation. In order to achieve this, we used the Monte Carlo radiative transfer code STOKES (Goosmann \& Gaskell 2007; Marin et al. 2012, 2015; Marin 2018) and pre-computed the ionization structure of the disc with the photoionization code TITAN (Dumont et al. 2003), suitable for hot optically thick media.

The plain spectral output of our Monte Carlo approach serves as a completely novel reflection spectral model that may be discussed with respect to the previous attempts by codes that aim for the same with different methods, typically solving the equations of radiative transfer. The polarization degree and angle computed upon the spectral results with STOKES then uniquely complement and improve the existing models with Chandrasekhar's formulae, which may also confirm correctness of the new simulation, if we demonstrate similar behaviour with respect to the main model parameters. Although the results presented in this paper do not represent full high-resolution tables, with the grid that was performed it is already possible to discuss important spectropolarimetric properties of the locally reflected radiation and to draw reasonable conclusions on the TITAN and STOKES codes' performance with respect to the other approaches mentioned.

Such analysis may then serve as a basis to the total spectropolarimetric X-ray models of AGNs that the authors plan to achieve in the near future using these local reflection computations. Contrarily to the mildly ( $\lesssim 10$ per cent) polarized primary radiation $(\mathrm{Be}-$ heshtipour et al. 2017; Tamborra et al. 2018; Beheshtipour 2018), the total polarization signal is expected to be significantly enhanced due to additional polarization signatures emerging from Compton scattering inside the illuminated disc (Schnittman \& Krolik 2009; Dovčiak et al. 2011). The total simulated spectra, possibly including also the returning radiation (Schnittman \& Krolik 2010; Taverna et al. 2020a), will then provide direct answer to the total polarization enhancement by reflection, which is physically well-reasoned to emerge, especially at hard X-rays. If the polarization induced by reflection pro $\mathrm{v}$ es to be important, the presented tables will also serve as an estimate tool for observational times needed by the upcoming $\mathrm{X}$-ray polarimetric missions for the faint AGN sources in the context of highly photon-demanding polarimetry (Fabiani \& Muleri 2014).

Recently, a similar attempt to compute polarization of thermal disc emission in stellar-mass systems including absorption effects was performed in Taverna et al. (2020b) with the codes CLOUDY (Ferland et al. 2013, 2017) and STOKES. Therefore, this work on reflection tables could also complement the ongoing research for XRBs, which are also on the target lists of IXPE or eXTP missions and where the polarization induced by reflection may also play an important role. In the near future, the presented local computations could serve as a foundation stone for physically consistent X-ray polarimetric data fitting of AGN or XRB sources.

The structure of this paper is as follows: Section 2 provides an overview of the physical model assumed, the numerical techniques performed, and the parametric grid that was used. In Section 3, we present the spectral and polarimetric properties of the new model. Strengths and weaknesses of the novel model and comparisons with similar approaches are further discussed in Section 4. In Section 5, we conclude our analysis and lay the ground for more extensive, future research.

\section{THE MODEL AND NUMERICAL IMPLEMENTATION}

\subsection{Physical model of the $\mathrm{BH}$ and its accreting structure}

The inner disc of a compact accreting source can be locally assumed as a plane-parallel slab with an electron scattering-dominated atmosphere (Shakura \& Sunyaev 1973; Novikov \& Thorne 1973). In the local co-moving frame of the disc, we approximate the otherwise geometrically thin disc as a semi-infinite optically thick slab with a constant density $n_{\mathrm{H}}=10^{15} \mathrm{~cm}^{-3}$. We stress that for acquiring accurate reflection spectra (for e.g. spectral line fitting), it is necessary to treat the stratified disc surface in full hydrostatic equilibrium, which causes thermal ionization instability and narrow two-phase zones in the medium between the disc and the corona (see e.g. Begelman et al. 
1983; Różańska \& Czerny 1996), even though the detailed geometry and boundary conditions of the illuminated disc atmosphere are unknown (Ballantyne et al. 2001; Ross \& Fabian 2005, 2007). It has been shown in the literature that constant-density models vary from those of hydrostatic equilibrium in emission line shapes, including the iron line complex around 6-7 keV, as well as in the continuum (Nayakshin et al. 2000; Nayakshin \& Kallman 2001; Péquignot et al. 2001; Ballantyne et al. 2001; Różańska et al. 2002; Dumont et al. 2002; Ross \& Fabian 2007; Różańska \& Madej 2008; Różańska et al. 2011; Vincent et al. 2016). The direct comparisons are not easy, since the ionization parameter has no meaning in the case of the hydrostatic equilibrium and it is also hard to define an average density (Różańska et al. 2002). Despite the ongoing discussion, our aim in this paper is to compute the polarization properties of the reflection in the same scenario that is usually used for interpreting the spectra of AGNs and XRBs. In the observed X-ray spectra of these objects, the reflection component is usually fitted with the models assuming a constant density slab. The difference between the reflection spectra for the constant density and hydrostatic equilibrium slabs would then be manifested in our polarization computations mainly through the polarization degree energy dependence, where the amount and shape of depolarization due to spectral lines would change.

We represent the proximity and luminosity of the primary powerlaw source by an ionization parameter in $\left[\mathrm{erg} \mathrm{cm} \mathrm{s}^{-1}\right]$ (see e.g. Tarter et al. 1969)

$\xi=\frac{4 \pi \int F_{E}(r) d E}{n_{\mathrm{H}}}$,

where $F_{E}(r) \sim E^{-\Gamma+1}$ is the radiation energy flux locally received on the surface of the disc, and the bounds of the integral follow the chosen high and low energetic cut-offs (see Section 2.2 for our selection). Cut-off on the hard-energy tail is motivated by inability of coronal electrons to gain speeds that would result in such hard emission in the estimated coronal conditions of $T \approx 10^{9} \mathrm{~K}$. Lowenergy cut-off is given by the energy of the seed thermal photons illuminating the corona, i.e. there are only few scattered photons with lower energy. For the case of our computations with STOKES, we will denote the ionization parameter as $\xi_{\mathrm{S}}$. The range of the $\xi_{\mathrm{S}}$ and $\Gamma$ parameters studied was motivated by observational and physical prospects as well as by easily achievable comparison with previously published models. We adopted the typical iron solar abundance from Asplund et al. (2005) with $A_{\mathrm{Fe}}=1.0$ (notation relative to the solar value), neglecting the presence of dust.

Our objective is first to pre-compute the vertically stratified ionization structure of the disc using isotropic illumination and then to continue with the 3D Monte Carlo polarization multiple-scattering simulation, incorporating appropriate line and continuum processes that take place in the disc. As we primarily focus on discs around supermassive BHs, we neglect in our X-ray band study the illumination from the thermal disc below the atmosphere. We neglect any possible primary radiation impact from the opposite side of the disc or self-irradiation by the disc in strong-gravity regime, and retain only the reflection effect of the power-law X-rays from the corona on top, which is expected to be Comptonized thermal radiation (Haardt 1993; Haardt \& Maraschi 1993; Schnittman \& Krolik 2010).

In order to efficiently use the local reflection tables in total emission computations in future works and to be able to discuss the effect of primary polarization degree and angle on the reflected emission, we assumed three distinct polarization states of the primary radiation for our runs: (a) unpolarized light with the normalized Stokes parameters ${ }^{1} Q_{\mathrm{P}}=0$ and $U_{\mathrm{P}}=0$, (b) horizontally polarized light with $Q_{\mathrm{P}}=-1$ and $U_{\mathrm{P}}=0$, (c) diagonally polarized light with $Q_{\mathrm{P}}=0$ and $U_{\mathrm{P}}=1$. The illumination and reflection from the slab was then studied for all possible incident $\mu_{\mathrm{i}}$ angles and emergent $\mu_{\mathrm{e}}, \Phi_{\mathrm{e}}$ angles. We denote $\mu_{\mathrm{i}}$ as the cosine of an angle measured from the disc's normal, and $\mu_{\mathrm{e}}$ as the cosine of an angle measured from the disc's normal. We denote $\Phi_{\mathrm{e}}$ as the relative azimuthal angle measured counterclockwise at the disc plane between the emission and the incident ray directions projected to the disc's surface [see e.g. fig. 1 in Dovčiak et al. (2011) for a sketch of the different local reflection angles].

\subsection{Numerical models}

\subsubsection{TITAN}

TITAN (Dumont et al. 2003) is a code designed for computing the radiative transfer together with the ionization structure of the optically thick media. In our application, we used the latest version of the code updated for many new atomic transitions (see Adhikari et al. (2015); Goosmann et al. (2016)). We use it to pre-compute the ionization structure, i.e. the fractional abundance of each element in different states of ionization inside a plane-parallel slab of gas in thermal and ionization equilibrium, isotropically illuminated by a given power-law primary spectrum from one side. The photoionization radiative transfer is done using the accelerated lambda iteration (ALI) method, while the ionization balance is computed in the full non-LTE approach, with iterations until the column depth $N_{\mathrm{H}}=10^{25} \mathrm{~cm}^{-2}$ that corresponds to the Thomson optical depth $\tau \sim 7$. TITAN solves the energy balance, the ionization, and the statistical equilibria, the transfer equations in a plane-parallel geometry, for the lines and continuum up to $26 \mathrm{keV}$, which is enough for the required output on line properties. For the purpose of this paper, we assume constant gas density over the slab with $n_{\mathrm{H}}=10^{15} \mathrm{~cm}^{-3}$. The primary photon-flux in TITAN has two exponential cut-offs and follows

$N(E)=N_{0} E^{-\Gamma} e^{-\frac{E}{E_{\mathrm{T}, \mathrm{c}}}} e^{-\frac{E_{\mathrm{T}, 0}}{E}}$

with much larger energy range between $E_{\mathrm{T}, 0}=0.03 \mathrm{keV}$, and $E_{\mathrm{T}, \mathrm{c}}=$ $300 \mathrm{keV}$. The output of TITAN is then processed by a specific $\mathrm{C}++$ script that produces a suitable input file for STOKES, containing all the ionization structure information.

\subsubsection{STOKES}

STOKES (Goosmann \& Gaskell 2007; Marin et al. 2012, 2015; Marin 2018) is a Monte Carlo radiative transfer code that was originally developed to solve near-IR to UV radiative transfer in $3 \mathrm{D}$ geometric shapes where scattering is the main source of opacity (as in, e.g. AGNs). In our case, we used the upgraded version 2.32 of STOKES, optimized for modelling of X-ray radiation, enabling reproduction of all 4 Stokes parameters $I, Q, U, V$ (in all the processes that we examine $V=0)$ for arbitrary incident polarization and $\left(\mu_{\mathrm{e}}, \Phi_{\mathrm{e}}\right)$ bins at the surface $(z=0)$ of the pre-computed plane-parallel slab with 50 vertical layers. The emission region, from which the photons are injected inside the slab, is located at $z=0$ and we prescribe also an incident angle $\mu_{\mathrm{i}}$ grid as one of the STOKES input parameters. No

1 The Stokes parameters $I, Q, U$ in all of this work correspond to their standard definitions in e.g. Chandrasekhar (1960) and the orientation convention in the polarization plane is further clarified in Section 3 with the definition of polarization angle. 
bulk motion is considered for the emission region. We use a primary photon-flux with the three distinct polarization states according to

$N(E)=N_{0} E^{-\Gamma}$,

where the cut-offs are sharp at $E_{\min }=10^{-1.1} \mathrm{keV}$ and $E_{\max }=$ $10^{2.4} \mathrm{keV}$. In order to guarantee similar amount of numeric noise across the energy range, we imposed that the photons are sampled equally in a logarithmically spaced grid in energy between $E_{\text {min }}$ and $E_{\max }(350$ bins with $\Delta \log E=0.01$ ) with weight assignment to each photon according to the power-law distribution, for which the results are compensated at the output. The energy resolution was motivated by reasonable computational times on one hand and on the other by ability to provide reasonable spectro-polarimetric analysis with respect to other known models and with respect to the past and forthcoming X-ray missions' resolution (IXPE, eXTP, XMM-Newton, ATHENA). The number of primary photons $N_{\text {tot }}$ per one simulation run varied with $\xi_{\mathrm{S}}, \Gamma$, and $\mu_{\mathrm{i}}$, but was always $\geq 3.5 \times 10^{8}$ with the aim to reduce numerical noise as much as possible within reasonable computational times.

During the simulation, photons are followed along their trajectory and experience multiple interactions resulting in the line and continuum spectral and polarization features, such as Compton downscattering, free-free interactions, photoelectric absorptions, etc. The STOKES 2.32 version used for our case did not account for (inverse) Compton up-scattering, nor synchrotron emission, which forms an acceptable compromise between complexity of the calculations and the energy range studied. A set of virtual detectors at the $\left(\mu_{\mathrm{e}}, \Phi_{\mathrm{e}}\right)$ bins subsequently registers all the photons that were not absorbed within the multiple-scattering region. At each detector, the Stokes parameters of the collected photons are summed after having rotated the Stokes parameter reference frames around the detector line of sight, to match with the detector frame. An auxiliary routine ANALYZE created by the authors of STOKES is used to process the results into conveniently readable text files. In the end, we receive the Stokes parameters with respect to a parametric grid of $E, \xi_{\mathrm{S}}, \Gamma, \mu_{\mathrm{i}}, \mu_{\mathrm{e}}, \Phi_{\mathrm{e}}$ for the unpolarized, horizontally, and diagonally polarized states of the primary radiation.

\subsubsection{Resulting FITS tables}

In order to provide the computations compactly and in a well arranged way to the user, we decided to create and attach FITS files (Hanisch et al. 2001) containing our results in reduced energy range and for 11 out of 20 originally computed $\mu_{\mathrm{e}}$ values between $\mu_{\mathrm{e} \text {, min }}=0.025$ and $\mu_{\mathrm{e}, \max }=0.975$ with $\Delta \mu_{\mathrm{e}}=0.05$. We preserve all the computed $\mu_{\mathrm{i}}$ with $\Delta \mu_{\mathrm{i}}=0.1$ and $\Phi_{\mathrm{e}}$ with $\Delta \Phi_{\mathrm{e}}=15^{\circ}$. The output Stokes parameters $I, Q$ and $U$, here below commonly denoted as $S$, were stored to the FITS files with the following normalization

$N\left(E ; \mu_{\mathrm{e}}, \mu_{\mathrm{i}}, \Phi_{\mathrm{e}}\right)=\frac{A \xi_{\mathrm{S}} n_{\mathrm{H}}}{4 \pi e \times 10^{10}} \frac{S\left(E ; \mu_{\mathrm{e}}, \mu_{\mathrm{i}}, \Phi_{\mathrm{e}}\right)}{N_{\mathrm{tot}} \Delta \mu_{\mathrm{e}} \Delta \Phi_{\mathrm{e}}}$,

where $e$ is the elementary charge in coulombs necessary for a unit conversion and

$$
A= \begin{cases}\frac{\left(E_{\max }^{1-\Gamma}-E_{\min }^{1-\Gamma}\right)(2-\Gamma)}{(1-\Gamma)\left(E_{\max }^{2-\Gamma}-E_{\min }^{2-\Gamma}\right)}, & \Gamma \notin\{1,2\}, \\ \ln \left(\frac{E_{\max }}{E_{\min }}\right) \frac{2-\Gamma}{E_{\max }^{2-\Gamma}-E_{\min }^{2-\Gamma},} & \Gamma=1, \\ \frac{E_{\max }^{1-\Gamma}-E_{\min }^{1-\Gamma}}{1-\Gamma} \ln ^{-1}\left(\frac{E_{\max }}{E_{\min }}\right), & \Gamma=2 .\end{cases}
$$

In this way, the photon-flux is stored in the units of [counts $\mathrm{cm}^{-2} \mathrm{~s}^{-1}$ ]. This normalization accounts for the differences in the cut-offs used
Table 1. Description of the attached FITS tables representing the reduced local reflection tables computed by STOKES.

\begin{tabular}{ll}
\hline \hline Number of tables & $\begin{array}{l}9 \text { (horizontal, diagonal, and no incident polarisation } \\
\text { versus } I, Q, U \text { output normalized according to (4)) }\end{array}$ \\
Spectral units & {$\left[\right.$ counts $\left.\mathrm{cm}^{-2} \mathrm{~s}^{-1}\right]$} \\
Energy range & $0.1 \mathrm{keV}$ to $100 \mathrm{keV}$ \\
Energy binning & $300 \mathrm{bins}, \Delta \log E=0.01$ \\
$\Gamma$ & $\{1.2,1.4,1.6,1.8,2.0,2.2,2.4,2.6,2.8,3.0\}$ \\
$\xi_{\mathrm{S}}$ index & $\{1,2,3,4,5,6,7,8,9,10,11\}$ \\
$\mu_{\mathrm{i}}$ & $\{0.0,0.1,0.2,0.3,0.4,0.5,0.6,0.7,0.8,0.9,1.0\}$ \\
$\mu_{\mathrm{e}}$ & $\{0.025,0.075,0.175,0.275,0.375,0.475,0.575$, \\
& $0.675,0.775,0.875,0.975\}$ \\
$\Phi_{\mathrm{e}}$ & $\left\{7.5^{\circ}, 22.5^{\circ}, 37.5^{\circ}, 52.5^{\circ}, 67.5^{\circ}, 82.5^{\circ}, 97.5^{\circ}\right.$, \\
& $112.5^{\circ}, 127.5^{\circ}, 142.5^{\circ}, 157.5^{\circ}, 172.5^{\circ}, 187.5^{\circ}$, \\
& $202.5^{\circ}, 217.5^{\circ}, 232.5^{\circ}, 247.5^{\circ}, 262.5^{\circ}, 277.5^{\circ}$, \\
Extensions & $\left.292.5^{\circ}, 307.5^{\circ}, 322.5^{\circ}, 337.5^{\circ}, 352.5^{\circ}\right\}$ \\
& Primary Header - description of the tables \\
& 'PARAMETERS' - parameter values \\
& 'ENERGIES' - low and high energetic bin edges \\
& 'SPECTRA' - values of the Stokes parameters and \\
& corresponding model parametric values \\
& 'XI(GAMMA)' - conversion table to real $\xi \mathrm{S}$ values \\
& depending on $\Gamma$ and $\xi \mathrm{S}$ index \\
\hline \hline
\end{tabular}

in TITAN and STOKES codes for the primary radiation and takes into consideration the units in standard output of the codes. In total, nine FITS files were created for the combinations of three incident polarization states with the three linear $(I, Q$ and $U$ ) Stokes parameters. For storage convenience, we reduce the energy range to 300 bins between 0.1 and $100 \mathrm{keV}$. The adopted $\xi_{\mathrm{S}}$ grid varies with $\Gamma$, but in general $\xi_{\mathrm{S}}$ spans values between 1.1 and 39225.5 . For this reason, we store index values $\{1,2, \ldots, 11\}$ instead of real $\xi_{\mathrm{S}}$ and attach a separate extension at the end of the FITS file, which holds the real $\xi_{\mathrm{S}}$ values assigned to indices for each $\Gamma$. Apart from this last extension, the attached FITS files conform to the OGIP standard for XSPEC (Arnaud 1995; Arnaud 1996) in this way. ${ }^{2}$ Table 1 brings a complete overview of the attached FITS files and adopted parametric mesh.

\section{RESULTS}

In all the spectral figures presented below, we display results per $\mathrm{keV}$ and use the normalization (4) or the following normalization in case of integrated STOKES spectra in angular space $\left(N_{\mu_{\mathrm{e}}}=20\right.$ in our case $)$

$N(E)=\frac{A \xi_{\mathrm{S}} n_{\mathrm{H}}}{N_{\mu_{\mathrm{e}}} 4 \pi^{2} e \times 10^{10}} \sum_{\mu_{\mathrm{e}}, \mu_{\mathrm{i}}, \Phi_{\mathrm{e}}} \mu_{\mathrm{i}} \Delta \mu_{\mathrm{i}} \frac{S\left(E ; \mu_{\mathrm{e}}, \mu_{\mathrm{i}}, \Phi_{\mathrm{e}}\right)}{N_{\text {tot }} \Delta \mu_{\mathrm{e}}}$,

if not mentioned otherwise. The linear polarization fraction $p$ and the polarization angle $\Psi$ are obtained from the STOKES output through the usual expressions

$\begin{aligned} p & =\frac{\sqrt{Q^{2}+U^{2}}}{I} \\ \Psi & =\frac{1}{2} \arctan _{2}\left(\frac{U}{Q}\right),\end{aligned}$

2 We are currently computing with the same method the full OGIP standard FITS file tables for XSPEC with the ionization parameter grid that does not depend on $\Gamma$. These tables will be published in the near future. 


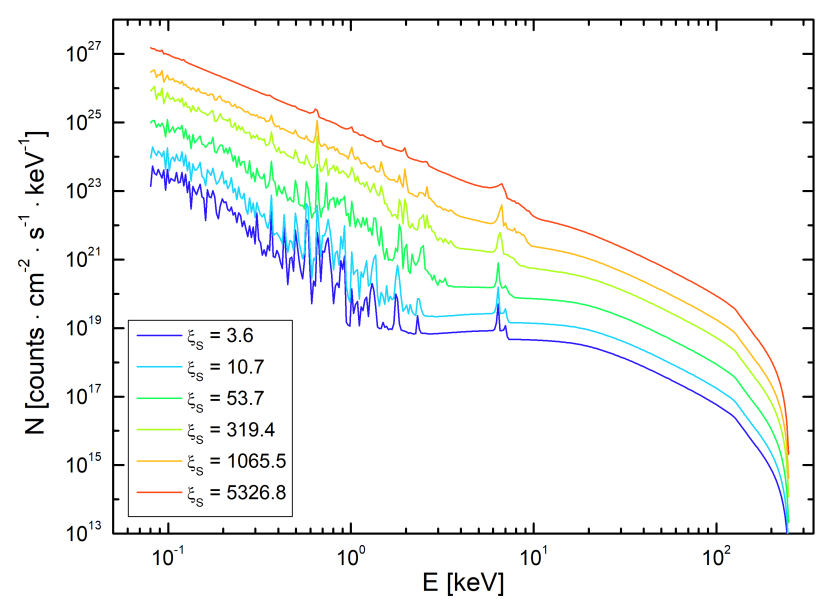

Figure 1. STOKES spectral results integrated in $\mu_{\mathrm{i}}, \mu_{\mathrm{e}}$ and $\Phi_{\mathrm{e}}$ for the unpolarized primary radiation, $\Gamma=2.2$. The color code corresponds to various $\xi_{\mathrm{s}}$.

where $\arctan _{2}$ denotes the quadrant-preserving inverse of a tangent function and $\Psi=0$ corresponds to a polarization vector oriented along the projected disc's normal to the polarization plane. $\Psi$ increases in the counterclockwise direction from the observer's point of view. Displays of polarization degree with energy have been averaged over the neighbouring 10 bins in energy [first $Q$ and $U$ in the output were averaged and then transformed via (7)] for the sake of numerical noise reduction but not to the detriment of physical information loss. But even this reduced resolution is far better than any X-ray polarimetric instrument planned for the IXPE (Weisskopf et al. 2013, 2016) or eXTP (Zhang et al. 2016, 2019) missions.

\subsection{Spectral properties}

We first show the X-ray spectral output $N(E)$ obtained by STOKES for initially unpolarized radiation (to be compared with XILLVER results in Section 4). Fig. 1 shows spectral results integrated in angular space according to (6), for various $\xi_{S}$ and one $\Gamma$. All the spectra possess characteristic features (see e.g. Fabian et al. 2000; Remillard \& McClintock 2006; Seward \& Charles 2010) such as the Compton hump at around $20 \mathrm{keV}$, a sharp decline at $E>10^{2} \mathrm{keV}$, an excess at soft energies, a forest of lines around $1 \mathrm{keV}$, and the most prominent $\mathrm{Fe} \mathrm{K} \alpha$ line at $6.4-7 \mathrm{keV}$, also confirmed by large number of observations from X-ray accreting sources (Gottwald et al. 1995; Winter et al. 2009; $\mathrm{Ng}$ et al. 2010). The expected result at energies above $10 \mathrm{keV}$ was achieved in STOKES, i.e. that Compton scattering, governed by the fairly constant Klein-Nishina cross-section with energy, is the dominant source of opacity at hard X-rays to the detriment of photoelectric absorption, which decays as $\sim E^{-3}$ (García et al. 2013). The behaviour of $N(E)$ with $\xi_{\mathrm{S}}$ corresponds to the cases in literature (see e.g. fig. 2 in Fabian et al. (2000)). Emission lines are superimposed to the continuum but become completely yielded once the ionization and reflection capabilities of the disc become higher and the re-processed radiation begins to resemble the shape of the original incident spectrum. Fabian et al. (2000) discusses four distinct regimes of spectral variation with $\xi$ based on atomic properties and absorption edges involved, which is supported by STOKES computations and the resulting Fig. 1.

A new result brought by our work is the role of incident polarization on the reflected spectral outcome. Although some X-ray polarization of the primary radiation is expected to be due to Comptonization of thermal radiation inside the corona, general estimates for the polarization degree of the primary source are rather low ( $\lesssim 10 \%)$ according to the recent analysis in Beheshtipour et al. (2017); Tamborra et al. (2018); Beheshtipour (2018). If some polarization was present, then, for symmetric reasons, vertically or horizontally polarized light should be dominant in case of extended coronal models (Dabrowski \& Lasenby 2001; Niedźwiecki \& Życki 2008; Schnittman \& Krolik 2010) at low heights. In case of lamp-post models (Matt et al. 1991; Martocchia \& Matt 1996; Henri \& Petrucci 1997; Martocchia et al. 2000; Dovčiak et al. 2004a; Miniutti \& Fabian 2004) generalrelativistic effects will rotate the polarization position angle along null geodesics from the corona towards the disc (Connors et al. 1980) and the situation becomes more general for incident disc irradiation, i.e. any incident state of polarization is possible. Our computations, apart from the unpolarized case, assumed two extreme cases of initial $p=100$ per cent polarization in order to estimate its possible effects in comparison with the completely unpolarized light and in order to test that the code adheres to the stated orientation conventions. Having appended these two initially polarized cases to our tables, it also allows for interpolation of reflection results for any input polarization state from this basis of the three computed independent polarization states, which will be necessary in future construction of global spectropolarimetric models. We aim to address any impact of light bending and other relativistic effects for a distant observer in our future works that will introduce the STOKES local tables integrated over the accretion disc with some adopted global geometry.

Typical effect of variable initial polarization on the local spectrum emitted in particular direction is shown on Fig. 2, which is about half order of magnitude in $E F=N E^{2}$ quantity (for $\Gamma=2$ to abstract from plain slope effects) in the Compton hump region. We checked multiple selected incident and emission angles and their spectra under different initial polarization. Despite the role of multiple scattering that depolarizes the incident radiation and smears the differences at the spectral output, the damping of the spectral output expected for single-scattered photons in certain angular configurations was confirmed (initially 100 per cent polarized light should be strongly filtered, if oriented perpendicular to the plane of scattering, upon Compton scattering close to the $\Theta=90^{\circ}$ scattering angle for single scatters). The amplitude of the spectral output was studied relative to other angular configurations. The maximum differences in the local reflected radiation for our three distinct initial polarization states achieve about an order of magnitude in $E F$ (for $\Gamma=2$ ) in the Compton hump region, but may also completely vanish in the spectral output. Variation of initial polarization (for any angular configuration) behaves at the spectral output in the same manner for different $\xi_{\mathrm{S}}$ and $\Gamma$, as there is no prominent connection of $\xi_{\mathrm{S}}$ and $\Gamma$ parameters with this effect induced by the most common single-scattering angle in the re-processed radiation.

If we integrate over incident and emergent angles and display the spectrum for the three cases of initial polarization studied, the differences are inconspicuous, as displayed on Fig. 3. Assuming an integration over angles with uniform weights to all computed incident and emission angles according to (6), we received average relative variation between the cases studied in the $E F=N E^{2}$ quantity of 0.3 per cent at $0.3-1 \mathrm{keV}, 2.5$ per cent at $2-10 \mathrm{keV}$, and 2.0 per cent at $11-15 \mathrm{keV}$. The insensitivity of the direction-averaged spectra to the incident polarization are due to almost even spanning of all dominant single-scattering angles $\Theta$. In our future works, we plan to address the non-uniform integration expected for realistic stronggravity environments. 


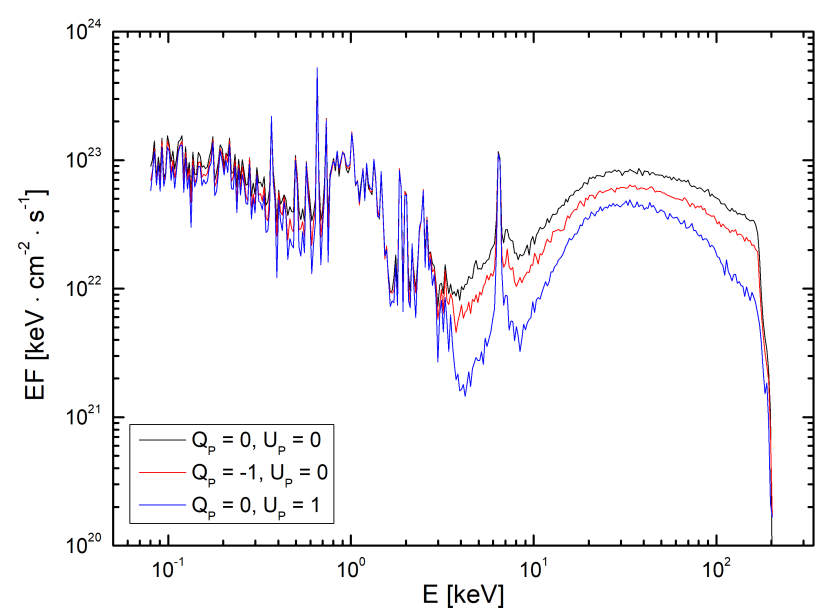

Figure 2. Directional spectrum obtained by STOKES for $\mu_{\mathrm{i}}=0.5, \mu_{\mathrm{e}}=0.475$, $\Phi_{\mathrm{e}}=292.5, \xi_{\mathrm{S}}=143.4$ and $\Gamma=2.0$. Three different primary polarizations are shown. Unpolarized light with $Q_{\mathrm{P}}=0$ and $U_{\mathrm{P}}=0$ (black), horizontally polarized light with $Q_{\mathrm{P}}=-1$ and $U_{\mathrm{P}}=0$ (red), and diagonally polarized light with $Q_{\mathrm{P}}=0$ and $U_{\mathrm{P}}=1$ (blue). This example represents typical differences in spectra for distinct primary polarization in the local reflection tables.

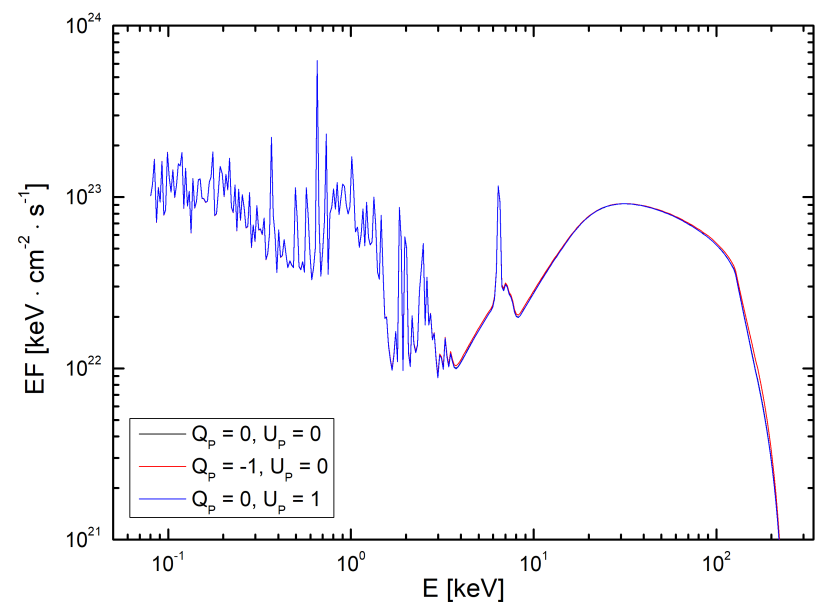

Figure 3. Spectrum obtained by STOKES integrated in $\mu_{\mathrm{i}}, \mu_{\mathrm{e}}$ and $\Phi_{\mathrm{e}}$ for $\xi_{\mathrm{S}}=143.4$ and $\Gamma=2.0$. Three different primary polarization states are shown. Unpolarized light with $Q_{\mathrm{P}}=0$ and $U_{\mathrm{P}}=0$ (black), horizontally polarized light with $Q_{\mathrm{P}}=-1$ and $U_{\mathrm{P}}=0$ (red), and diagonally polarized light with $Q_{\mathrm{P}}=0$ and $U_{\mathrm{P}}=1$ (blue).

Even though Fig. 2 suggests that variable primary polarization may play a role for an amplitude of hard X-ray spectra in some strongly directional global GR configurations, one needs to realize that we show initially 100 per cent polarized light versus an unpolarized case, while coronal studies suggest low estimates on the primary polarization degree. Therefore, we still remain sceptical about any seeing of such spectral effects (either on the continuum or the $\mathrm{Fe} \mathrm{K} \alpha$ line) in realistic global models. However, if one brings attention to the polarization output itself, i.e. the polarization degree and angle, which will be soon measured by the forthcoming X-ray polarimetric missions, the primary polarization will play a more significant role in the reflected light (see Section 3.2).

Strength of the reflected radiation with respect to the model param-

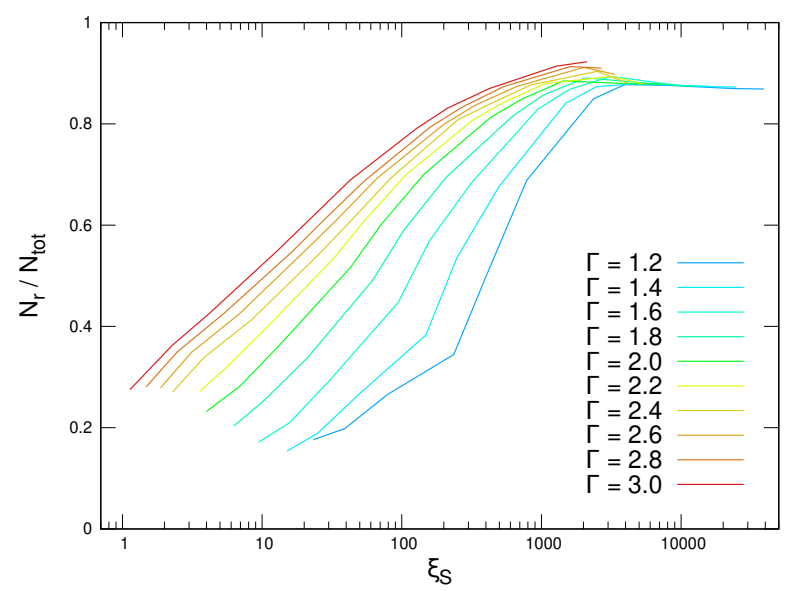

Figure 4. Total reflectivity obtained by STOKES as it varies with $\xi_{\mathrm{S}}$ for different $\Gamma$, integrated over the total energy range and over all $\mu_{\mathrm{i}}$.

eter values can be obtained by displaying the total disc reflectivity, i.e. the quantity $N_{\mathrm{r}} / N_{\text {tot }}$, where $N_{\mathrm{r}}$ denotes the number of photons detected in the STOKES output for unpolarized primary radiation with a given $\mu_{\mathrm{i}}, \xi_{\mathrm{S}}$, and $\Gamma$, integrated in specific energy range and in all emission directions (i.e. in the entire $\mu_{\mathrm{e}}$ and $\Phi_{\mathrm{e}}$ angular space). This quantity will generally behave differently at soft X-rays, hard X-rays, and if integrated in the total energy range. None the less, we found that reflectivity slowly declines with increasing $\mu_{\mathrm{i}}$ in the soft, hard, and total energy range, regardless of $\xi_{\mathrm{S}}$ and $\Gamma$ choice. This tendency is natural, because more absorption is expected for high $\mu_{\mathrm{i}}$ as the incident photons will penetrate further below the disc's surface in a more vertical incident ray direction. Since this tendency is similar for all parametric combinations, it is possible to further integrate disc reflectivity in $\mu_{\mathrm{i}}$, in order to obtain better statistics on its behaviour with respect to $\xi_{\mathrm{S}}$ and $\Gamma$ at different energy bands.

In Fig. 4, we show the reflectivity with respect to $\xi_{\mathrm{S}}$ for various $\Gamma$ cases, integrated over the full energy range and over all $\mu_{\mathrm{i}}$. The fact that reflectivity rises with increasing $\xi_{\mathrm{S}}$ is related to Fig. 1, which already confirmed that the disc tends to act more like a mirror of the primary illumination for higher ionization. Furthermore, harder Xray illumination, i.e. lower $\Gamma$ parameter, yields lower total reflection, but for completely ionized discs there is no difference. The quantitative estimates of the total disc reflectivity may be important e.g. for thermal reverberation in the accretion disc, where it is assumed that a fraction of the illuminating coronal X-rays are absorbed and thus the disc is heated (see, e.g. Kammoun et al. 2019; Dovčiak et al. 2021).

\subsection{Polarization properties}

The polarization properties of light will change when scattered of free electrons, or when line transitions are involved. Recombination lines come out unpolarized, because they statistically even out, even if single emission was polarized. Resonant lines and Compton scattering involves incoming photon with arbitrary polarization that is redistributed with polarization properties, which are ultimately governed by scattering angle, having the most effective polarization change for $\Theta \approx 90^{\circ}$. Therefore, in the first place and for single-scattered photons, geometrical discussion should focus upon the incident and emergent angles $\mu_{\mathrm{i}}, \mu_{\mathrm{e}}$, and $\Phi_{\mathrm{e}}$, which can altogether form right angles in numerous ways. Multiple scattering, which is more likely for larger $\mu_{\mathrm{i}}$, always distorts this dominant effect for single-scattered photons. 


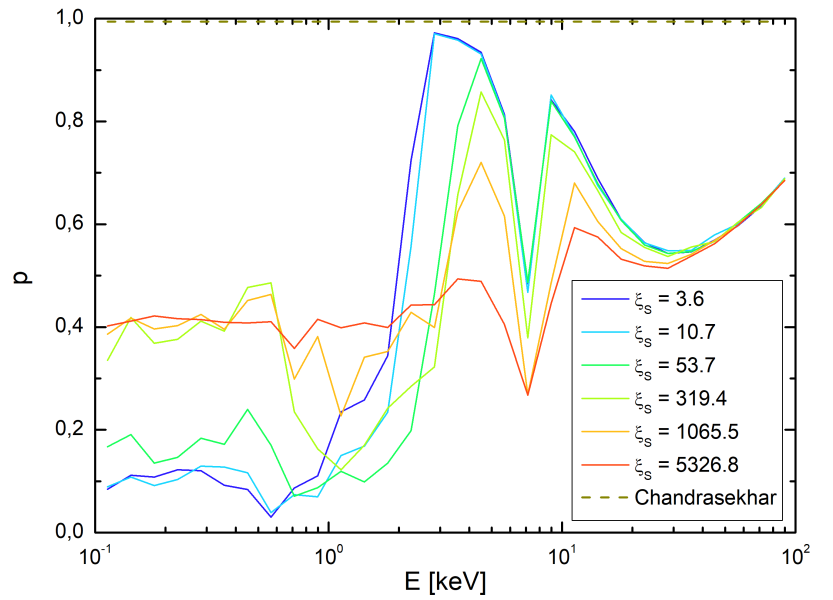

Figure 5. The polarisation degree obtained by STOKES (solid lines) for the unpolarized primary radiation, $\mu_{\mathrm{i}}=0.5, \mu_{\mathrm{e}}=0.475, \Phi_{\mathrm{e}}=292.5, \Gamma=2.2$, and varying $\xi_{\mathrm{S}}$. Value for the single-scattering approximation by Chandrasekhar's formulae for unpolarized primary radiation in this geometry is shown (dashed line, hardly visible on the top of the figure).

Then, in the second place, we should reflect on energetic properties of possible (de)polarization and the energy-dependent cross-sections of these processes, including absorption of a photon. This is also coupled to geometry, as for different $\mu_{\mathrm{i}}$ various depths are likely to be reached and the cross-sections of line and continuum processes vary with the disc's vertical stratification. Lastly, photons may change energy due to Compton down-scattering and one photon may encounter multiple kind of processes with different treatment of polarization during its journey through the medium. None the less, some distinct polarization features in locally reflected radiation still appear in the final output of the STOKES simulations. They can be unambiguously explained by one or two origins.

Regarding the polarization degree $p$, Fig. 5 displays variation of $p(E)$ with $\xi_{\mathrm{S}}$ for unpolarized primary radiation and fixed generic set of remaining parameters. Fig. 6 displays also the non-integrated $p(E)$ values for a similar set of parameters, but with varying initial polarization. Fig. 6 uses the same set of model parameters as in Fig. 2 and demonstrates higher relative impact of incident polarization conditions on $p$ than on the spectral output. It was already clear from Figs 2 and 3 that, at the soft part of the spectrum below $3 \mathrm{keV}$, where most spectral lines are present, the differences with respect to the initial polarization states are minor. Figs 5 and 6 confirm that spectral lines act destructively on any non-zero incident polarization and that the lines manifest through low $p$ at soft X-rays, and with a low dip in $p$ at the $\mathrm{Fe} \mathrm{K} \alpha$ line at $6.4-7 \mathrm{keV}$. Although some polarization would be expected from resonant lines, they do not appear dominant in the output, and may be also impacted by multiple scattering, which distorts any clear single scattering contribution on overall polarization state.

A tendency of rising polarization degree towards higher energies is visible, as less lines are present in the hard X-rays and pure Compton recoil (which is a dominant process for polarization emergence in our set-up) prevails. A large dip similar to the mirrored Compton hump shape in $N(E)$ is always present around $20 \mathrm{keV}$. This is expected, as multiple scattering happens in the Compton hump energy band, to which the photons from the primary radiation are largely redistributed in energy. Hence, a decrease in polarization degree emerges over a generally large polarization degree in the hard X-rays, which are

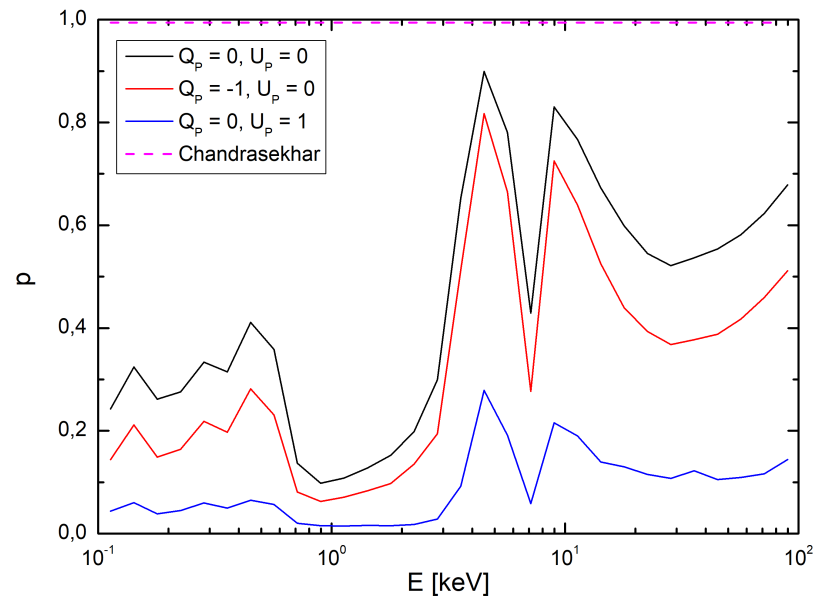

Figure 6. The polarization degree obtained by STOKES (solid lines) for STOKES (solid lines) for $\mu_{\mathrm{i}}=0.5, \mu_{\mathrm{e}}=0.475, \Phi_{\mathrm{e}}=292.5, \xi_{\mathrm{S}}=143.4$ and $\Gamma=2.0$. Three different primary polarizations are shown. Unpolarized light with $Q_{\mathrm{P}}=0$ and $U_{\mathrm{P}}=0$ (black), horizontally polarized light with $Q_{\mathrm{P}}=-1$ and $U_{\mathrm{P}}=0$ (red), and diagonally polarized light with $Q_{\mathrm{P}}=0$ and $U_{\mathrm{P}}=1$ (blue). This example corresponds to the parametrization shown in Fig. 2. Value for the single-scattering approximation by Chandrasekhar's formulae for unpolarized primary radiation in this geometry is shown (dashed line, hardly visible on the top of the figure).

not affected by spectral lines. On Fig. 5, strong variations with $\xi_{\mathrm{S}}$ are less detectable in the high-energy tail, as none of the Stokes parameters varies with $\xi_{\mathrm{S}}$ there. In the low-energy tail, polarization rises with increasing $\xi_{\mathrm{S}}$ due to gradually less spectral lines being present (see Fig. 1). In the soft energy band, regarding the definition of $p(7)$, the rise of intensity with $\xi_{\mathrm{S}}$ is compensated by even larger rise in $Q$ and $U$, while the slopes originating in primary power-law cancel out. Antagonistic trend around $10 \mathrm{keV}$ cannot be explained by spectral lines presence and is due to natural rise of intensity (still observable on Fig. 1 to the detriment of $Q$ and $U$ increment at these energies). The fact that absorption of photons (see Fig. 1) may result in enhanced polarization degree was already discussed in Taverna et al. (2020b).

In order to study further effects with more clarity, we averaged $p$ over $E>10 \mathrm{keV}$, where Compton scattering is by far the most important re-processing mechanism, and selected generic $\Gamma$ and $\xi_{\mathrm{S}}$. $p\left(\Phi_{\mathrm{e}}\right)$ is displayed on Fig. 7 for various $\mu_{\mathrm{i}}$ and $\mu_{\mathrm{e}}$ values. In this way, we may study the angular behaviour of polarization output with respect to the dominant single-scattering angle in STOKES simulations and compare them to the Chandrasekhar's formulae (Chandrasekhar 1960) for Rayleigh single scattering of unpolarized incident radiation inside a horizontal slab. These analytical values were already implemented to the previously mentioned graphs of $p$, but with little importance, as their values were overly high for the selected geometries, although it can be concluded that unpolarized incident radiation reached these idealized values around $3 \mathrm{keV}$. Fig. 7 clearly displays the code's results with respect to the analytical approach in the reflection scenario. STOKES treats the scattering angles (via $\mu_{\mathrm{i}}, \mu_{\mathrm{e}}$ and $\Phi_{\mathrm{e}}$, which together set the dominant single-scattering angle) with precision and the polarization degree results are bilaterally symmetric in $\Phi_{\mathrm{e}}$.

The presence of multiple scattering and general crudeness of the Chandrasekhar's approximation, unsuitable for reflection processes on the accretion discs of AGNs (further discussed in Section 4), are 


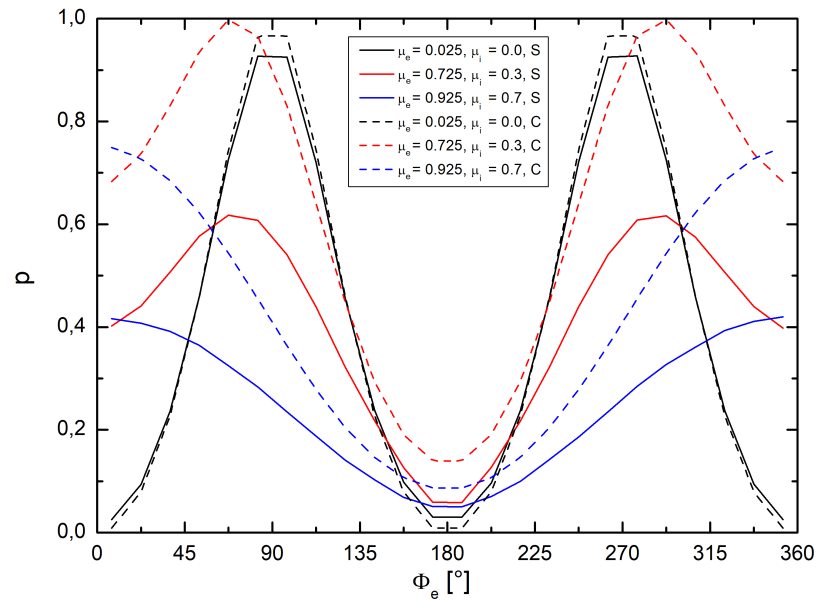

Figure 7. The polarisation degree versus azimuthal emission angle obtained by STOKES (solid lines) integrated at $E>10 \mathrm{keV}$ for unpolarized primary radiation, $\xi_{\mathrm{S}}=106.5, \Gamma=2.2$, and various $\mu_{\mathrm{i}}, \mu_{\mathrm{e}}$ combinations. Values for the single-scattering approximation by Chandrasekhar's formulae for unpolarized primary radiation in these geometries are shown (dashed lines).

the reasons for the lack of $p$ in the STOKES semirealistic simulation behind the idealized analytical values. To further prove the effect of multiple scatterings, we put a condition in the STOKES routines for only one electron scattering event per photon to mimic the singlescattering analytical approach in the Monte Carlo code. We tested two examples on Fig. 7, where the dashed and solid lines, i.e. the analytical and numerical approach, differ significantly: (a) $\Phi_{\mathrm{e}}=$ $67.5^{\circ}, \mu_{\mathrm{e}}=0.725, \mu_{\mathrm{i}}=0.3$, the red lines, and (b) $\Phi_{\mathrm{e}}=67.5^{\circ}$, $\mu_{\mathrm{e}}=0.925, \mu_{\mathrm{i}}=0.7$, the blue lines; rest of the parameters remain as for Fig. 7. The average polarization degree above $10 \mathrm{keV}$ in this approach was (a) 98.08 per cent (for the Chandrasekhar's singlescattering approximation this is 99.77 per cent, for the STOKES full multiple-scattering simulation this is 61.74 per cent) and (b) 53.12 per cent (for the Chandrasekhar's single-scattering approximation this is 54.37 per cent, for the STOKES full multiple-scattering simulation this is 32.50 per cent).

The polarization angle tends to oscillate around a constant value at all energies in the spectral lines, i.e. these oscillations are rather visible at the soft X-rays for lower $\xi_{S}$ values, as far as our adopted energy resolution allows to investigate. Apart from degenerate coordinate cases (because of presence of the so-called critical point in the definition of polarization angle discussed in, e.g. Dovčiak et al. (2011), when the polarization plane is perpendicular to the disc's normal) or low polarization degree, where the most frequent value is ambiguous due to low statistics, the polarization angle of the continuum is otherwise constant in energy (apparent when one e.g. averages over the 10 neighbouring energy bins), because $Q$ and $U$ tends to behave in the same manner with $E$. This implies independence of the polarization angle of the continuum on $\Gamma$, and the independence of the polarization angle of the continuum on $\xi_{\mathrm{S}}$ is also observed. If we construct the polarization angle quantity from data integrated in all incident and emission angles, for the purpose of obtaining larger photon statistics, we observe $\Psi \approx 90^{\circ}$ at all energies (by $\lesssim 2^{\circ}$ variation in the total range), apart from a few remaining spectral lines that tend to manifest as single points in energy in our resolution around $90^{\circ} \pm 90^{\circ}$.

Since the average value of $\Psi$ over the 10 neighbouring energy bins does not depend on $E, \Gamma$ and $\xi_{\mathrm{S}}$, it is also reasonable to integrate over

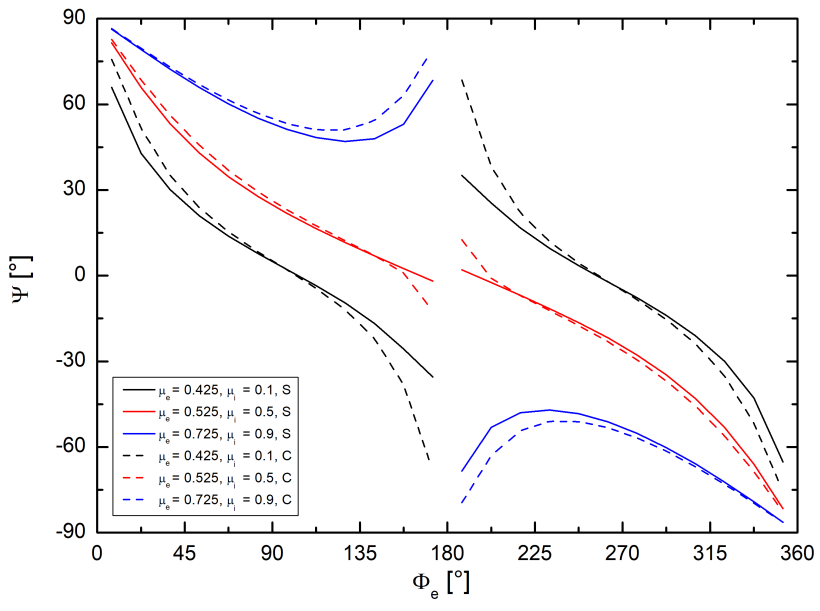

Figure 8. The polarisation angle versus azimuthal emission angle obtained by STOKES (solid lines) integrated in $E, \Gamma$, and $\xi_{\mathrm{S}}$ for the unpolarized primary radiation and various $\mu_{\mathrm{i}}, \mu_{\mathrm{e}}$ combinations. Values for the single-scattering approximation by Chandrasekhar's formulae for unpolarized primary radiation in these geometries are shown (dashed lines).

these parameters in order to obtain better statistics on its geometrical behaviour without loss of generality. Similarly to Fig. 7, Fig. 8 displays $\Psi\left(\Phi_{\mathrm{e}}\right)$ for various $\mu_{\mathrm{i}}$ and $\mu_{\mathrm{e}}$ values and in comparison to the same Chandrasekhar's formulae for Rayleigh single-scattering of unpolarized incident radiation. Likewise, we obtained results close to the analytical approximation also for the cases of horizontal and diagonal primary polarization, which we omit here for brevity, and all of these figures can be explained by the role of scattering angle in polarization change upon single scattering, despite some distortion caused by multiple scattering and despite some numerical noise. STOKES is again very precise in treating the scattering angle geometry with respect to the polarization vector located in the polarization plane, and the obtained polarization angle values follow the analytical behaviour quite nicely notwithstanding the fact that the $\Psi$ quantity, given its definition, in addition suffers from coordinate degeneracy.

\section{DISCUSSION}

\subsection{Other spectral reflection models}

The spectral part of the TITAN and STOKES results obtained by us can be directly compared to similar attempts in the literature, unlike our polarization results, which are unique. The problematic of reflection spectra from accretion discs was already addressed by e.g. the computations with NOAR (Dumont et al. 2000) and PEXRAV (Magdziarz \& Zdziarski 1995). These models assumed a neutral disc for simplicity, which produces a considerable flux deficiency at soft X-rays that is not supported by the expected temperatures that impose complicated structures in terms of ionization. PEXRIV (Magdziarz \& Zdziarski 1995 ) is another model already involving an ionized disc, but being too simple in assuming isothermal medium with a maximum temperature of $T=10^{6} \mathrm{~K}$. Another attempt for a reflection code involving ionized disc was completed byRoss \& Fabian (1993); Ross et al. (1999); Ross \& Fabian (2005) with the REFLIONX models. The most recent major step further was achieved by García et al. (2013), where the ionization structure up to optical depth $\sim 10$, was computed by the XSTAR code (Kallman \& Bautista 2001), and spectral reflec- 
tion tables were subsequently computed using the Feautrier radiative transfer solving method (Mihalas 1978), resulting in the XILLVER tables (García \& Kallman 2010; García et al. 2011; García et al. 2013). The paper by García et al. (2013) also introduces a discussion and comparison of these up-to-date most precise spectral reflection tables in X-rays with computations using the older REFLIONX tables that utilize Fokker-Planck diffusion equation, including a modified Kompaneets operator (Ross et al. 1978; Ross 1979), and that also already assume an ionized structure, but e.g. underestimate the amount of neutral atoms (García et al. 2013). ${ }^{3}$

In the next subsection, our contribution to this problem will be the presentation of a comparison of the combined TITAN and STOKES local spectral tables in the reflection scenario with the REFLIONX tables and XILLVER tables that are the most widely used models by the scientific community today and that greatly resemble our setup. In the future, we plan to compare our local results with the XILLVER results in the global view, i.e. as if the inner accretion system was observed by a distant observer, including the primary source.

\subsection{Direct comparison of our spectral results with the XILLVER and REFLIONX tables}

The XILLVER reflection spectra, which are also available in the FITS format (García et al. 2013), assume unpolarized primary radiation and are integrated in $\mu_{\mathrm{i}}$ and $\Phi_{\mathrm{e}}$ parameters. As spectral dependency on $\mu_{\mathrm{e}}$ in terms of shape and amplitude is not dramatic, it is reasonable to average XILLVER tables in all $\mu_{\mathrm{e}}$ in order to obtain better statistics and confine to the average $\mu_{\mathrm{e}}=0.5$ value, which is also an average inclination angle for STOKES in our mesh set-up. Comparison of the STOKES spectral results under unpolarized incident illumination with XILLVER tables is shown on Fig. 9. For this comparison, the raw STOKES output $I$ was averaged and normalized such as

$N(E)=\frac{\xi_{\mathrm{S}} n_{\mathrm{H}} \int_{E_{\min }}^{E_{\max }} E^{-\Gamma} d E}{B N_{\mu_{\mathrm{e}}} 4 \pi^{2} e \times 10^{10}} \sum_{\mu_{\mathrm{e}}, \mu_{\mathrm{i}}, \Phi_{\mathrm{e}}} \mu_{\mathrm{i}} \Delta \mu_{\mathrm{i}} \frac{I\left(E ; \mu_{\mathrm{e}}, \mu_{\mathrm{i}}, \Phi_{\mathrm{e}}\right)}{N_{\mathrm{tot}} \Delta \mu_{\mathrm{e}} \Delta E}$,

where

$B=E_{\mathrm{X}, \mathrm{c}}^{2-\Gamma}\left[\Gamma_{\mathrm{f}}\left(2-\Gamma, \frac{E_{\mathrm{X}, 0}}{E_{\mathrm{X}, \mathrm{c}}}\right)-\Gamma_{\mathrm{f}}\left(2-\Gamma, \frac{E_{\mathrm{X}, 1}}{E_{\mathrm{X}, \mathrm{c}}}\right)\right]$,

and

$\Gamma_{\mathrm{f}}(s, x)=\int_{x}^{\infty} t^{s-1} \mathrm{e}^{-t} d t$

is the upper incomplete gamma function and $E_{\mathrm{X}, 0}=0.1 \mathrm{keV}, E_{\mathrm{X}, 1}=$ $1000 \mathrm{keV}, E_{\mathrm{X}, \mathrm{c}}=300 \mathrm{keV}$ are values related to the used XILLVER cut-offs. The normalization (8) differs from (6), because apart from the units and geometrical conventions, it is necessary to again account for alternative cut-offs used in XILLVER tables as opposed to the STOKES computations. Namely, the high-energy cut-off in XILLVER is exponential and was selected as $E_{\mathrm{X}, \mathrm{c}}=300 \mathrm{keV}$ (XILLVER tables offer to choose $E_{\mathrm{X}, \mathrm{c}}$ as a free model parameter) and low-energy cutoff is sharp at $E_{\mathrm{X}, 0}=0.1 \mathrm{keV}$. Hence, the primary radiation there follows equation

$N(E)=N_{0, \mathrm{X}} E^{-\Gamma} e^{-\frac{E}{E_{\mathrm{X}, \mathrm{c}}}}$,

\footnotetext{
3 All the differences between REFLIONX and XILLVER on the spectral output are still not fully understood (García et al. 2013).
}

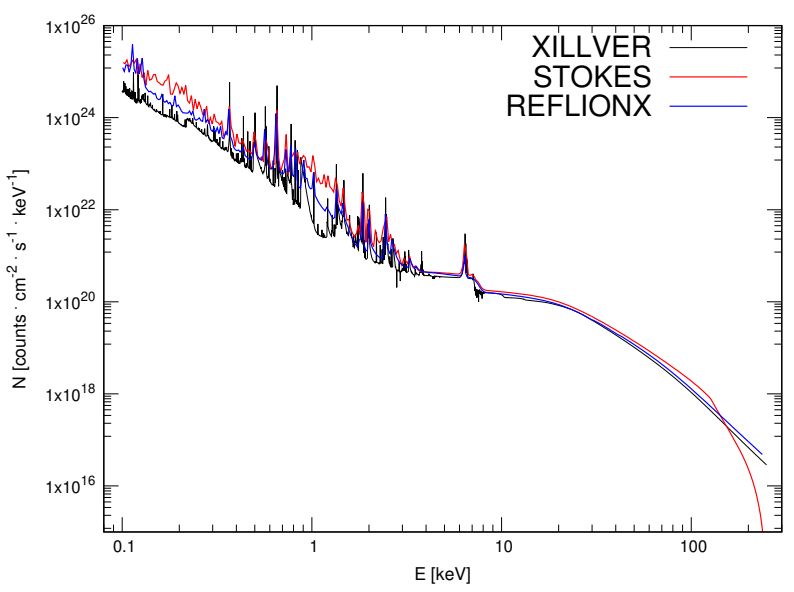

Figure 9. Comparison of the XILLVER tables (black) for $\xi_{X}=100$ and $\Gamma=2.2$, and REFLIONX tables (blue) for $\xi_{\mathrm{R}}=100$ and $\Gamma=2.2$ with the corresponding STOKES spectral results (red) for $\xi_{\mathrm{S}}=106.5$ and $\Gamma=2.2$, all normalized accordingly.

where $N_{0, \mathrm{X}}$ corresponds to $\xi_{\mathrm{X}}$, which we further use to denote the ionization parameter in XILLVER grid, via

$\xi_{\mathrm{X}}=\frac{4 \pi N_{0, \mathrm{X}}}{n_{\mathrm{H}}} B$.

In STOKES, both cut-offs are sharp and primary radiation follows equation (3). In Fig. 9, the original XILLVER tables are in addition divided by $\Delta E$ at each bin and multiplied by the necessary factor $\frac{10^{20}}{4 \pi} \mu_{\mathrm{e}}$ that accounts for FITS storage convention and geometrical definitions therein. Last but not least, we also multiplied the XILLVER tables by $\xi_{\mathrm{S}} / \xi_{\mathrm{X}}$ factor to compensate for the differences in $\xi$, as this parameter does not have the same grid in both computations and directly determines the amplitude at all energies. In order to compare both models efficiently, we selected the closest $\xi_{\mathrm{S}}$ parameter in STOKES tables to our $\xi_{\mathrm{X}}$ selection. XILLVER uses similar grid in $\Gamma$ parameter and the same values could be used for comparison without a need of interpolation. The same holds for elemental abundance $A_{\mathrm{Fe}}=1.0$ present in both tables, but we note that the solar chemical composition used in XILLVER (from Grevesse \& Sauval 1998) is severely outdated with respect to the one used in STOKES (from Asplund et al. 2005), see the discussion in Grevesse et al. (2007).

At the Compton hump energies $(\sim 20 \mathrm{keV})$, both spectra remarkably match with the adopted normalization. This holds similarly for other comparable ionization parameters and photon-indices, if displayed, as Fig. 9 is only an example of such comparison. We stress this conclusion, since our tables were obtained by a completely different (Monte Carlo) method, while XILLVER tables were obtained by simultaneously solving the equations of radiative transfer, energy balance, and ionization equilibrium (also in a Compton-thick, planeparallel medium), using the Feautrier method (García \& Kallman 2010; García et al. 2011; García et al. 2013)). Therefore, our local results are cross-checked in the hard X-rays before further use in, e.g. total spectral production of AGNs, or before further exploration of our technique.

Juxtaposition of models at these energies makes most sense, because $\xi$ variation and subsequent compensation for uneven $\xi$ grid does not permeate heavily at these energies (see e.g. Fig. 1), although the uneven grid in $\xi$ is believed to be the biggest issue in setting the normalization accordingly and inherently leaves discrep- 
ancies in amplitude. Also, line production based on different atomic data produced by TITAN and XSTAR is not present at this range.

In García et al. (2013), the strong influence of cut-off definition chosen in the primary power law on the spectral shape, which also has to be taken into account when comparing two different codes, was pointed out. In particular, García et al. (2013) illustrates the impact of different low-energy (continuous and broken power law) and highenergy (exponentials with different $E_{\mathrm{X}, \mathrm{c}}$ ) cut-offs on the reflected local spectrum acquired in XILLVER. This manifests itself especially at energies below $1 \mathrm{keV}$. The spectral differences due to alternative cut-off shapes and positions can reach one order of magnitude. The change in overall spectrum is stronger, if higher $\Gamma$ is used (see figs 1 and 2 in García et al. 2013). This is inherently connected to the temperature profile with optical depth $\tau$ inside the disc. For our case, the temperatures computed by TITAN stay in between the profiles of XILLVER (provided for similar parametric set-up in García et al. (2013)) until $\tau \sim 1$ but in deeper layers the disc remain about an order of magnitude hotter in TITAN. Also, our computations ended at layers with $\tau \sim 7$, which is less by 3 than the depths used in radiative transfer computations of XILLVER tables, which may be another reason behind the discrepancies below $1 \mathrm{keV}$. The fact that XILLVER and STOKES results vary in some line features and their strength is not surprising, as different atomic data are used, our computations include $\sim 4000$ atomic transitions, which is nearly half of the spectral lines that are considered in XSTAR, and for constructing XILLVER models calculations of level populations, temperature, total opacity, and emissivity are performed by XSTAR, not TITAN.

In the attempts of comparing XILLVER results to REFLIONX, PEXRAV or PEXRIV tables in García et al. (2013), the differences also mostly appeared in the soft energy range. To contribute to this discussion, we displayed the corresponding REFLIONX computations along our comparisons between STOKES and XILLVER. The REFLIONX tables (Ross \& Fabian 1993; Ross et al. 1999; Ross \& Fabian 2005) are stored in the same FITS format as the XILLVER tables and contain the same high- and low-energy cut-offs. Thus, we again choose the closest ionization parameter $\xi_{\mathrm{R}}$ to our $\xi_{\mathrm{X}}$ and $\xi_{\mathrm{S}}$ selection, the same power-law index $\Gamma$ and the same elemental abundance $A_{\mathrm{Fe}}=1.0$ (but now with a solar chemical composition from Morrison \& McCammon 1983) and we normalize REFLIONX in the same way as XILLVER with an additional factor of 2, which accounts for the fact that the REFLIONX tables are already averaged in $\mu_{\mathrm{e}}$. We conclude that at soft X-rays, where most discrepancies appear, the STOKES models are typically closer to the REFLIONX computations, rather than the XILLVER computations. An example is Fig. 9, where the REFLIONX spectrum is added. A more detailed comparison of the soft X-ray results obtained by STOKES and other computational methods is required to understand the apparent differences in the soft X-ray range. We plan to do this in future work. Here, we focus on the X-ray polarization results that are not sensitive to the spectral discrepancies around $1 \mathrm{keV}$.

Weakness of our spectral model compared to the XILLVER computations is the neglect of Compton up-scattering in the disc matter in STOKES code. We may also include an extra thermal component of the X-ray radiation originating from the disc and synchrotron processes to our model in the future that would make the STOKES X-ray tables more applicable to XRBs and more precise in the soft X-rays. A floating grid in primary cut-off boundaries, non-constant density, or variable elemental abundance is also something that can be implemented in the future versions of our models and so far has not been tested due to large computational times. Our primary objective was to timely deliver the first very realistic polarization computations in the reflection scenario for the upcoming X-ray polarimetric missions that operate at hard X-rays above $2 \mathrm{keV}$.

Because STOKES accounts for multiple scattering, it is almost impossible to give a quantitative insight of the importance of inverse Compton scattering and synchrotron emission. Howver, qualitatively, inverse Compton scattering will likely smooth the high-energy tail of the Compton hump due to energy shifts (García et al. 2020) and decrease a little bit the polarization degree of our current results. In general, inverse Compton scatterings tend to reduce the polarization degree of emission; multiple Compton scatterings do so even more (Krawczynski 2011). Unpolarized synchrotron photons give rise to synchrotron self-Compton emission with vanishing or very small $(<5$ per cent) polarization degrees for all but very low Lorentz factors (Celotti \& Matt 1994; Begelman \& Sikora 1987). What is interesting is that the inverse Compton emission should track the polarization degree and polarization direction of the synchrotron emission. All of this is of prime importance for radio-loud objects. Our paper focuses on radio-quiet sources, so at first order we can safely neglect those effects (synchrotron emission in particular, which is in addition more relevant to optically thin media, rather than to optically thick standard disc), but we admit that inverse Compton scattering should be included in future simulations.

An advantage of our spectra over the XILLVER tables is the model dependency on $\mu_{\mathrm{i}}$ and $\Phi_{\mathrm{e}}$ angles, which is more demanding on the total number of photons $N_{\text {tot }}$ for the Monte Carlo method on one hand, but allows higher geometrical precision and angular checks that involve macroscopic analysis of scattering processes in the disc medium. Angular precision in the scattering simulation is especially crucial for the polarization outcome, as the creation/alteration of polarization is undoubtedly connected to (a)symmetries in the origin of radiation and accurate geometrical shapes. So far we have illuminated our disc slab with isotropic radiation for the pre-computations with TITAN but here we could also acquire higher precision in the future with incident inclination-dependent computations of ionization structure. An advantage of our new model worth mentioning is that incident arbitrary polarization of the primary radiation is now enabled, which is of course more important in the context of polarimetry, as it was already discussed in Section 3. In the future, we plan to develop tables with a fixed grid in ionization parameter that would also have a much higher resolution in energy. Such tables would enable further discussion upon spectral line features and disc structure modelling, although they might not be of any use to the low-resolution and photon-demanding X-ray polarimetry.

\subsection{Further validation of our polarization results and implications}

Despite some weaknesses of our current model, the spectral comparisons already validate a discussion of polarization of the reflected emission obtained uniquely by STOKES in the energy ranges of the forthcoming polarimetric missions IXPE or eXTP $(\gtrsim 2 \mathrm{keV})$ and further in the hard X-rays. Moreover, Fig. 5 showed generally higher predicted polarization fraction above $2 \mathrm{keV}$ than in the soft X-rays due to the polarization induced by Compton down-scattering in the disc. Although this is promising for targeting AGN and XRB sources by the IXPE or eXTP missions, the total polarization measured by a distant observer is expected to be lower due to the impact of integration over the disc (even though in some specific configurations the polarisation degree can be magnified due to general-relativistic effects, see Dovčiak et al. 2011) and the impact of direct primary radiation (expected to be mildly polarized, $\lesssim 10$ per cent, see e.g. 
Tamborra et al. 2018; Beheshtipour et al. 2017; Beheshtipour 2018) reaching the observer.

In Fig. 7, we already showed that the polarization degree predicted by our semirealistic simulation is lower than the analytical formulae forecast due the presence of multiple scattering and general crudeness of the Chandrasekhar's approximation. The Chandrasekhar's formulae represent a single-scattering approximation of elastic Rayleigh scattering without the ability to reconstruct characteristic Compton recoil around $20 \mathrm{keV}$ or the effects caused by spectral lines. None the less, they confirm the output of our simulation with respect to basic angular parameters and this approximation may also confirm basic energy dependence of the polarization quantities, especially the expected tendency of $p$ to rise towards harder X-rays, in global models once we apply them to the new local reflection tables (see e.g. Dovčiak et al. 2011).

\section{CONCLUSIONS}

In this paper, we discussed a method to simulate spectrum and polarization of locally reflected $\mathrm{X}$-ray radiation from $\mathrm{BH}$ accretion discs. We chose a power-law primary source that represents a hot gaseous corona situated above a semi-infinite disc slab. The ionization structure of the disc was pre-computed by a radiative transfer code TITAN (Dumont et al. 2003), suitable for ionized optically thick media. The low-resolution reflection tables comprising all linear $(I, Q$ and $U$ ) Stokes parameters were computed by a Monte Carlo code STOKES (Goosmann \& Gaskell 2007; Marin et al. 2012, 2015; Marin 2018) that incorporates important X-ray line and continuum mechanisms, including the characteristic multiple Compton down-scattering of $\mathrm{X}$-ray photons in the partially ionized disc medium. We computed the energy-dependent X-ray outcome, i.e. the Stokes parameters $I$, $Q$ and $U$, for three arbitrary polarization states of the primary radiation, numerous ionization parameters $\xi_{\mathrm{S}}$, photon-indices $\Gamma$, incident inclinations $\mu_{\mathrm{i}}$, and emission angles $\mu_{\mathrm{e}}$ and $\Phi_{\mathrm{e}}$. The results are made available to the reader in a compact form of the FITS format in the attachment of this paper. The new simulation can be methodically compared to spectral reflection models of similar kind appearing in the literature and it represents a unique and first attempt to numerically compute X-ray polarization in the local reflection scenario.

We showed that with all the model assumptions and physical processes incorporated in TITAN and STOKES we were able to produce $\mathrm{X}$-ray spectra possessing the most common reflection features supported by up-to-date theoretical, numerical, and observational information on radio-quiet $\mathrm{X}$-ray compact accreting sources, such as the Compton hump at around $20 \mathrm{keV}$, a sharp decline at $E>10^{2} \mathrm{keV}$, an excess at soft energies, a forest of lines around $1 \mathrm{keV}$, and the most prominent $\mathrm{Fe} \mathrm{K} \alpha$ line at $6.4-7 \mathrm{keV}$. The ionization parameter, defined by (1), correctly enforces stronger reflection with larger illumination and the reflected spectrum starts to resemble the original power-law shape at high $\xi_{\mathrm{S}}$ values. We found that extreme initial polarization of the primary radiation may affect significantly the spectral output in selected geometries, but if integrated over the incident and emission angles, which can imitate a subsequent integration over the disc for total spectral production (as the source would be observed from infinity), we acquired almost identical spectra.

We managed to validate our method by successful normalization and comparison to the up-to-date most widely used reflection spectral tables obtained by XSTAR called XILLVER (Kallman \& Bautista 2001; García \& Kallman 2010; García et al. 2011; García et al. 2013), as well as the older REFLIONX reflection spectra (Ross \& Fabian 1993; Ross et al. 1999; Ross \& Fabian 2005). The comparison was achieved with similar model parameters and complete angular integration of these models. The spectra appeared very similar above $3 \mathrm{keV}$, which is positive for the $2-12 \mathrm{keV}$ energy range that will be explored by the upcoming polarimetric missions, for which we primarily develop our spectropolarimetric models. The remaining discrepancies, especially in the soft X-rays, may lie in the uneven $\xi$ grid between the tables, different atomic data, adopted optical depths, selected cut-offs of the primary radiation, or the computational methods themselves, especially at the disc structure numerical stage. In the future, we may improve our STOKES local simulation by incorporating Compton upscattering, and disc black-body radiation and include more flexibility in the disc's density, elemental abundance, or primary cut-off selection.

From our results we may already discuss the polarization properties of locally reflected X-ray radiation. We showed that reflection can locally induce large polarization degrees despite multiple scattering and other depolarization factors. Comparing our models to the available Chandrasekhar's analytical approximation of single scattering in a slab region (Chandrasekhar 1960), we confirmed the expected behaviour of polarization degree and angle with respect to the incident and emission angles in the local co-moving frame. We confirmed the relative importance of the initial polarization state of the primary radiation that imprints the polarized outputs. In the crude resolution of $\Delta \log E=0.1$, the local polarization angle remains independent of $E, \Gamma$, and $\xi_{\mathrm{S}}$ and variations of the local polarization degree with $\xi_{\mathrm{S}}$ are prominent in the whole energy range. In the soft part of the spectrum, the polarization degree increases with ionization to the detriment of general depolarization caused by spectral lines, while in the medium-to-hard energy range (between 2 and $30 \mathrm{keV}$ ) it decreases with ionization, probably due to lower absorption (larger absorption induces also larger polarization degree in the X-ray thermal emission in case of accretion discs in X-ray binaries studied by Taverna et al. 2020b). The fact that the polarization degree of the reflected X-ray emission can be, locally, quite large (i.e. tens of per cents) between 2 and $12 \mathrm{keV}$, is a good sign for the forthcoming X-ray polarimetric missions that operate at these frequencies.

With these low-resolution tables, we may already produce some total spectra and discuss the global spectropolarimetric properties of $\mathrm{BH}$ accreting sources, as well as compute more realistic estimates of observational times needed for the forthcoming X-ray polarimetric missions. This will be properly addressed in our future works. It has been argued already in the introduction that reflection models are an important part of consistent modeling of compact radio-quiet accreting sources. We believe that the presented approach may bring fresh insights into the X-ray spectral modelling of inner accretion phenomena and that the polarization tables achieved in this way will later become a valuable component for the total emission models and for data fitting of AGN and XRB sources that will soon be needed for the X-ray polarimetric mission IXPE (Weisskopf et al. 2013, 2016), due to be launched in 2021, and that may become valuable in terms of constraining inclination and orientation of real sources, $\mathrm{BH}$ spins, coronal morphologies or disc's structures.

\section{ACKNOWLEDGEMENTS}

MD thanks for the support from the GACR project 21-06825X and the institutional support from RVO:67985815. JP acknowledges financial support from the Charles University, project GA UK no. 174121, and from the Barrande Fellowship Programme of the Czech and French governments. AR was supported by Polish National Science Center grant no. 2015/17/B/ST9/03422. 


\section{DATA AVAILABILITY}

The model reflection tables underlying this article (created and compiled by authors of this article) are available in the FITS format in the Figshare Repository at https://doi.org/10.6084/m9. figshare. 16726207.

\section{REFERENCES}

Abramowicz M. A., Fragile P. C., 2013, Living Rev. Relativity, 16, 1 Adhikari T. P., Różańska A., Sobolewska M., Czerny B., 2015, ApJ, 815, 83 Antonucci R., 1993, ARA\&A, 31, 473

Arnaud K. A., 1995, in Office of Guest InvestigatorPrograms Memo OGIP/92009. NASA Goddard Space Flight Center, Laboratory for High Energy Astrophysics

Arnaud K. A., 1996, in Jacoby G. H., Barnes J., eds, Astronomical Society of the Pacific Conference Series Vol. 101, Astronomical Data Analysis Software and Systems V. p. 17

Asplund M., Grevesse N., Sauval A. J., 2005, in Barnes Thomas G. I., Bash F. N., eds, Astronomical Society of the Pacific Conference Series Vol. 336, Cosmic Abundances as Records of Stellar Evolution and Nucleosynthesis. p. 25

Ballantyne D., Ross R., Fabian A., 2001, MNRAS, 327, 10

Begelman M. C., Sikora M., 1987, ApJ, 322, 650

Begelman M. C., McKee C. F., Shields G. A., 1983, ApJ, 271, 70

Beheshtipour B., 2018, PhD thesis, Washington University in St. Louis

Beheshtipour B., Krawczynski H., Malzac J., 2017, ApJ, 850, 14

Celotti A., Matt G., 1994, MNRAS, 268, 451

Chandrasekhar S., 1960, Radiative Transfer. Dover Publications, New York

Compère G., Oliveri R., 2017, MNRAS, 468, 4351

Connors P. A., Stark R. F., 1977, Nature, 269, 128

Connors P. A., Piran T., Stark R. F., 1980, ApJ, 235, 224

Dabrowski Y., Lasenby A. N., 2001, MNRAS, 321, 605

Done C., Gierliński M., Kubota A., 2007, A\&ARv, 15, 1

Dovčiak M., Karas V., Yaqoob T., 2004a, ApJ, Supplement Series, 153, 205

Dovčiak M., Karas V., Matt G., 2004b, MNRAS, 355, 1005

Dovčiak M., Muleri F., Goosmann R. W., Karas V., Matt G., 2008, MNRAS, 391, 32

Dovčiak M., Muleri F., Goosmann R. W., Karas V., Matt G., 2011, ApJ, 731, 75

Dove J. B., Wilms J., Maisack M., Begelman M. C., 1997, ApJ, 487, 759

Dovčiak M., Papadakis I. E., Kammoun E. S., Zhang W., 2021, arXiv e-prints, p. arXiv:2110.01249

Dumont A.-M., Abrassart A., Collin S., 2000, A\&A, 357, 823

Dumont A.-M., Czerny B., Collin S., Zycki P. T., 2002, A\&A, 387, 63

Dumont A.-M., Collin S., Paletou F., Coupé S., Godet O., Pelat D., 2003, A\&A, 407, 13

Fabian A. C., Iwasawa K., Reynolds C. S., Young A. J., 2000, PASP, 112, 1145

Fabiani S., Muleri F., 2014, Astronomical X-Ray Polarimetry. Aracne, Roma

Ferland G. J., et al., 2013, Rev. Mex. Astron. Astrofis., 49, 137

Ferland G. J., et al., 2017, Rev. Mex. Astron. Astrofis., 53, 385

García J., Dauser T., Reynolds C. S., Kallman T. R., McClintock J. E., Wilms J., Eikmann W., 2013, ApJ, 768, 146

García J. A., Sokolova-Lapa E., Dauser T., Madej J., Różańska A., Majczyna A., Harrison F. A., Wilms J., 2020, ApJ, 897, 67

García J., Kallman T. R., 2010, ApJ, 718, 695

García J., Kallman T. R., Mushotzky R. F., 2011, ApJ, 731, 131

Goosmann R. W., Gaskell C. M., 2007, A\&A, 465, 129

Goosmann R. W., Holczer T., Mouchet M., Dumont A. M., Behar E., Godet O., Gonçalves A. C., Kaspi S., 2016, A\&A, 589, A76

Gottwald M., Parmar A. N., Reynolds A. P., White N. E., Peacock A., Taylor B. G., 1995, A\&AS, 109, 9

Grevesse N., Sauval A. J., 1998, Space Sci. Rev., 85, 161

Grevesse N., Asplund M., Sauval A. J., 2007, Space Sci. Rev., 130, 105

Haardt F., 1993, ApJ, 413, 680

Haardt F., Maraschi L., 1993, ApJ, 413, 507
Hanisch R., Farris A., Greisen E., Pence W., Schlesinger B., Teuben P., Thompson R., Warnock A., 2001, A\&A, 376

Henri G., Petrucci P. O., 1997, A\&A, 326, 87

Kallman T., Bautista M., 2001, ApJ, Supplement Series, 133, 221

Kammoun E. S., Papadakis I. E., Dovčiak M., 2019, ApJ, 879, L24

Krawczynski H., 2011, ApJ, 744, 30

Krolik J. H., 1999, Active galactic nuclei : from the central black hole to the galactic environment. Princeton University Press, Princeton, NJ

Kubota A., Done C., 2018, MNRAS, 480, 1247

Li L.-X., Narayan R., McClintock J. E., 2009, ApJ, 691, 847

Magdziarz P., Zdziarski A. A., 1995, MNRAS, 273, 837

Marin F., 2014, MNRAS, 441, 551

Marin F., 2016, MNRAS, 460, 3679

Marin F., 2018, A\&A, 615, A171

Marin F., Goosmann R. W., Gaskell C. M., Porquet D., Dovčiak M., 2012, A\&A, 548, A121

Marin F., Goosmann R. W., Gaskell C. M., 2015, A\&A, 577, A66

Martocchia A., Matt G., 1996, MNRAS, 282, L53

Martocchia A., Karas V., Matt G., 2000, MNRAS, 312, 817

Matt G., Perola G. C., Piro L., 1991, A\&A, 247, 25

Matt G., Fabian A. C., Ross R. R., 1993, MNRAS, 264, 839

Mihalas D., 1978, Stellar Atmospheres. 2nd edition, San Francisco: W.H. Freeman

Miniutti G., Fabian A. C., 2004, MNRAS, 349, 1435

Morrison R., McCammon D., 1983, ApJ, 270, 119

Nayakshin S., Kallman T. R., 2001, ApJ, 546, 406

Nayakshin S., Kazanas D., Kallman T. R., 2000, ApJ, 537, 833

Netzer H., 2015, ARA\&A, 53, 365

Ng C., Díaz Trigo M., Cadolle Bel M., Migliari S., 2010, A\&A, 522, A96

Niedźwiecki A., Życki P. T., 2008, MNRAS, 386, 759

Novikov I. D., Thorne K. S., 1973, in Black Holes (Les Astres Occlus), ed. C. DeWitt and B. S. DeWitt. Gordon and Breach, Paris, pp 343-450

Péquignot D., et al., 2001, in Ferland G., Savin D. W., eds, Astronomical Society of the Pacific Conference Series Vol. 247, Spectroscopic Challenges of Photoionized Plasmas. p. 533

Remillard R. A., McClintock J. E., 2006, ARA\&A, 44, 49

Reynolds C. S., 2019, Nature Astron., 3, 41

Reynolds C. S., Nowak M. A., 2003, Physics Reports, 377, 389

Ross R. R., 1979, ApJ, 233, 334

Ross R. R., Fabian A. C., 1993, MNRAS, 261, 74

Ross R. R., Fabian A. C., 2005, MNRAS, 358, 211

Ross R. R., Fabian A. C., 2007, MNRAS, 381, 1697

Ross R. R., Weaver R., McCray R., 1978, ApJ, 219, 292

Ross R. R., Fabian A. C., Young A. J., 1999, MNRAS, 306, 461

Różańska A., Czerny B., 1996, Acta Astron., 46, 233

Różańska A., Madej J., 2008, MNRAS, 386, 1872

Różańska A., Dumont A. M., Czerny B., Collin S., 2002, MNRAS, 332, 799

Różańska A., Madej J., Konorski P., Sadowski A., 2011, A\&A, 527, A47

Schnittman J. D., Krolik J. H., 2009, ApJ, 701, 1175

Schnittman J. D., Krolik J. H., 2010, ApJ, 712, 908

Seward F. D., Charles P. A., 2010, Exploring the X-ray Universe. Cambridge University Press, New York

Shakura N. I., Sunyaev R. A., 1973, A\&A, 24, 337

Stark R. F., Connors P. A., 1977, Nature, 266, 429

Tamborra F., Matt G., Bianchi S., Dovčiak M., 2018, A\&A, 619, A105

Tarter C. B., Tucker W. H., Salpeter E. E., 1969, ApJ, 156, 943

Taverna R., Zhang W., Dovčiak M., Bianchi S., Bursa M., Karas V., Matt G., 2020a, MNRAS, 493, 4960

Taverna R., Marra L., Bianchi S., Dovčiak M., Goosmann R., Marin F., Matt G., Zhang W., 2020b, MNRAS, 501, 3393

Trümper J. E., Hasinger G., 2008, The Universe in X-Rays. Springer-Verlag Berlin Heidelberg

Vincent F. H., Różańska A., Zdziarski A. A., Madej J., 2016, A\&A, 590, A132

Weisskopf M. C., et al., 2013, in Siegmund O. H., ed., Society of PhotoOptical Instrumentation Engineers (SPIE) Conference Series Vol. 8859, UV, X-Ray, and Gamma-Ray Space Instrumentation for Astronomy XVIII. p. 885908, doi:10.1117/12.2024473 
Weisskopf M. C., et al., 2016, The Imaging X-ray Polarimetry Explorer (IXPE). Proceedings of the SPIE, doi:10.1117/12.2235240

Winter L. M., Mushotzky R. F., Reynolds C. S., Tueller J., 2009, ApJ, 690, 1322

Zhang S. N., et al., 2016, Space Telescopes and Instrumentation 2016: Ultraviolet to Gamma Ray

Zhang S.-N., et al., 2019, Sci. China Phys. Mech. Astron., 62, 29502

This paper has been typeset from a $\mathrm{T}_{\mathrm{E}} \mathrm{X} / \mathrm{L} \mathrm{LT} \mathrm{E} \mathrm{X}$ file prepared by the author. 